\title{
Experimentando con Arduino y Scilab: propagación de calor en una barra metálica
}

Experimenting with Arduino and Scilab: heat propagation in a metal bar

\author{
E. Buksman*1@, A. L. Fonseca de Oliveira $^{1}$, L. Barbieri $^{1}$, C. Ferreira ${ }^{1}$ \\ ${ }^{1}$ Universidad ORT Uruguay, Facultad de Ingeniería, Montevideo, Uruguay
}

Recibida en 24 de December, 2018. Revisado en 3 de Mayo, 2019. Aceptado en 14 de Mayo, 2019.

\begin{abstract}
Este artículo presenta un dispositivo flexible y de bajo costo equipo diseñado para la realización de experiencias en el área de propagación de calor, el cual está basado en tecnología Arduino y el ambiente gráfico Xcos de Scilab. Además de la ventaja pedagógica de la implementación realizada por estudiantes de grado de ingeniería, la utilización del equipo ha posibilitado, mediante resultados experimentales, la determinación del coeficiente de convección y el análisis, y ajuste, del efecto de los sensores utilizados en la medida.
\end{abstract}

Palabras clave: Propagación de calor, Convección de calor, Arduino, Scilab.

This article presents a low cost multipurpose device based in Arduino technology and Xcos-Scilab graphical environement, designed for heat propagation experiences. In addition to the pedagogical advantage of the implementation carried out by engineering degree students, the use of the equipment has enabled, through experimental results, the determination of the convection coefficient and the analysis, and adjustment, of the effect of the sensors used in the measurement.

Keywords: Heat propagation, Heat convection, Arduino, Scilab.

\section{Introducción}

En los últimos años ha habido una expansión en el uso de tecnologías accesibles de bajos costo que posibilitan la realización de experimentos en diversas áreas.

La placa Arduino Uno [1] es una placa electrónica económica de uso genérico, que posibilita el control y la adquisición de datos, ideal para ser usada en como parte de dispositivos de laboratorio de Física e Ingeniería [2] [3] [4] 5].

En este artículo se presenta un dispositivo construido con bajo presupuesto (menos de cincuenta dólares americanos) que utiliza elementos de fácil adquisición como a ser caja plástica, placa Arduino Uno, sensores de temperatura, barra de aluminio, madera, cables, etc. La implementación del sistema formó parte de un proyecto realizado por estudiantes de ingeniería, con la supervisión de profesores, con la idea de ser utilizado como parte experimental en diferentes cursos como ser Calor y Fluidos, Teoría Ondulatoria, Sistemas de Control, etc. Este dispositivo permite medir, y controlar, la temperatura en tiempo real en una barra metálica.

Prototipos utilizando similar tecnología han sido utilizados por varios autores para el estudio del transporte de calor en barras metálicas. En 6] se estudia la propagación de calor, tomando en cuenta le convección, para una entrada de temperatura constante (baja temperatura con

${ }^{*}$ Correo electrónico: buksman@ort.edu.uy respecto al ambiente), siendo que en otros trabajos 7] [8], la barra es aislada de manera que la convección sea irrelevante, o esta es despreciada frente al transporte de calor por conducción. En este artículo se amplia el estudio tomando en cuenta el efecto de la convección para una entrada constante de temperatura, así como la propagación de una onda de calor para una entrada sinusoidal.

La estructura de este artículo es como se presenta a continuación.

En la sección 2 se detalla la construcción del equipo que contiene la barra de aluminio y el control de calentamiento de la misma, así como el ambiente gráfico de programación utilizado.

El análisis teórico de la propagación de calor en una barra con calentamiento constante o en forma sinusoidal es presentado en la sección 3. Considerando un balance energético y usando la ecuación de Fourier de conducción de calor, junto a la ecuación de convección, se presentan soluciones analíticas en régimen permanente. Como forma de aproximar las ecuaciones de calor en régimen transitorio, se utiliza una analogía eléctrica del transporte de calor compuesta por una red de resistencias y capacitores (analogía RC).

En la sección 4 se realiza un ajuste a las curvas experimentales de forma a determina el coeficiente de convección $h$. El efecto de la utilización de elementos externos a la teoría (sensores) ocasiona perturbaciones en los valores experimentales esperados respecto a la teoría. El modelo 
$\mathrm{RC}$ posibilita realizar correcciones locales en el modelo de forma a que los transitorios de las temperaturas se ajusten a los datos experimentales. Finalmente, con este modelo se analiza el régimen sinusoidal para todo punto de la barra.

En la conclusiones, sección 5 se presenta un resumen de los resultados obtenidos.

\section{Equipo}

\subsection{Placa electrónica, software y sensores}

La placa Arduino Uno [1] es una placa electrónica de uso genérico y de bajo costo, para la cual existen diversos ambientes públicos de programación [1] [9] [10]. Diseñada originalmente por el Interaction Design Institute (Ivrea, Italia), se ha propagado en diferentes versiones y ha sido utilizada en diversos proyectos de educación científica 11] 12. Para las placas de la familia Arduino existen varias extensiones llamadas 'shields', que amplían la funcionalidad del dispositivo mediante sensores $\mathrm{y} / \mathrm{u}$ otros. En este trabajo se utiliza el ambiente de programación estándar Arduino IDE (utiliza lenguaje similar a $\mathrm{C}++$ ) que puede ser descargado en www.arduino.cc.

Para el control de la placa y visualización de los datos se utiliza el ambiente gráfico Xcos del programa Scilab. Scilab 13 es un programa público para cálculo numérico ampliamente utilizado en ciencia e ingeniería, en aplicaciones tan diversas como: procesamiento de señal, cálculos estadísticos, optimización y control automático. El ambiente gráfico Xcos es un ambiente de programación visual mediante bloques funcionales que permiten un rápido desarrollo. Mediante el toolbox Arduino for $X \cos 14$ es posible la comunicación serial vía puerto USB con la placa, posibilitando la utilización del Arduino como una placa de control y adquisición de datos.

El montaje del sistema completo es ilustrado en la Figura 1.

En la construcción se ha utilizado una barra metálica de aluminio con forma de prisma rectangular de largo $L=$ $109,0 \mathrm{~mm}$, ancho $l=12,0 \mathrm{~mm}$ y espesor $e=2,0 \mathrm{~mm}$. Los sensores de temperatura utilizados en los experimentos

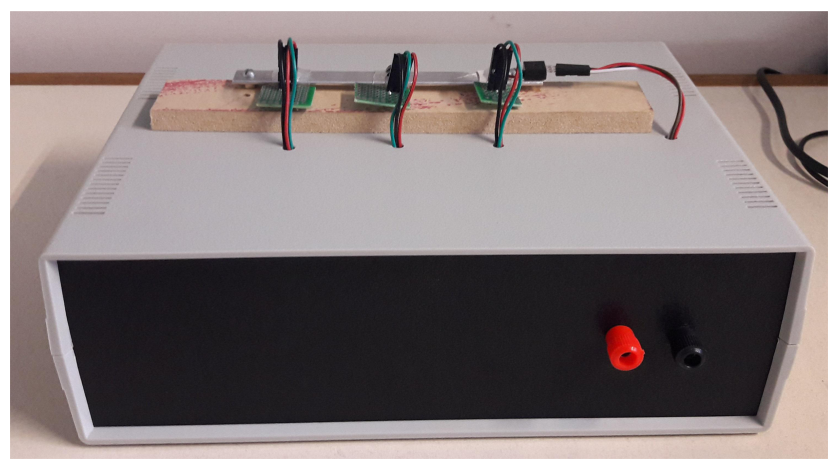

Figura 1: Equipo. son del tipo LM35 de Texas Instruments. Estos detalles son ilustrados en la Figura 2

El calor en la barra es generado mediante la disipación de un transistor del tipo TIP41C que trabaja en corte y zona activa controlado por un puerto con salida PWM del Arduino. La Figura 3 muestra el circuito de comando del transistor mediante un opto-acoplador que protege la salida de la placa.

Utilizando en el puerto de salida del $X \cos$ un valor entre 0 y 255 se controla el ciclo de trabajo de la señal PWM que es generada en la salida del Arduino. El esquema del circuito es representado en la Figura 4.

La alimentación de los sensores es realizada mediante la fuente de $5 \mathrm{~V}$ existente en las placas Arduino. El circuito del transistor es alimentado por una fuente externa de $16 \mathrm{~V}$.

\subsection{Procedimiento del experimento}

En esta sección se muestra el procedimiento estándar de utilización del equipo, explicado a continuación (ejemplo indicado en la figura).

- Se abre la interfaz del ambiente de desarrollo (Arduino IDE) y se selecciona el puerto COM apropiado (puerto USB que se ha conectado la placa).

- Se abre el archivo toolbox_arduino_v3.ino que pertenece al toolbox Arduino for Xcos. El código del archivo debe ser compilado y descargado en la placa.

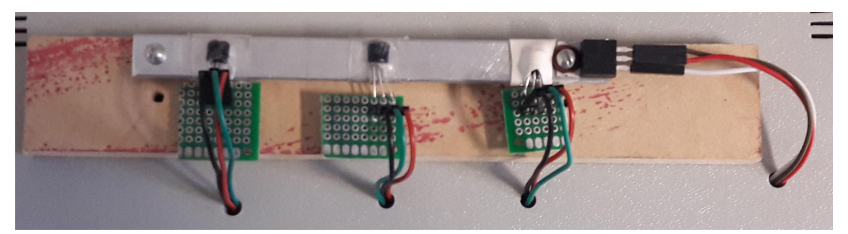

Figura 2: Detalle de la barra y sensores.

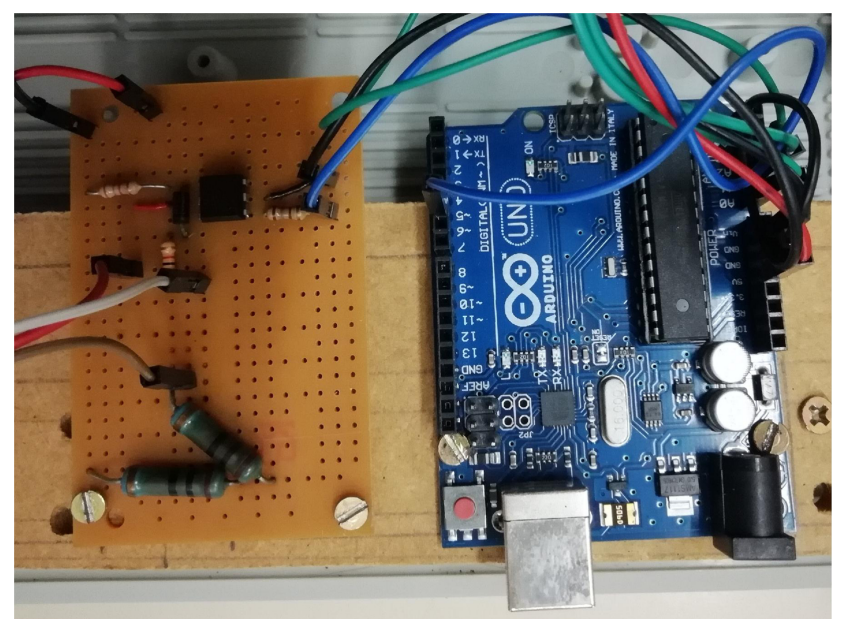

Figura 3: Caja abierta. 


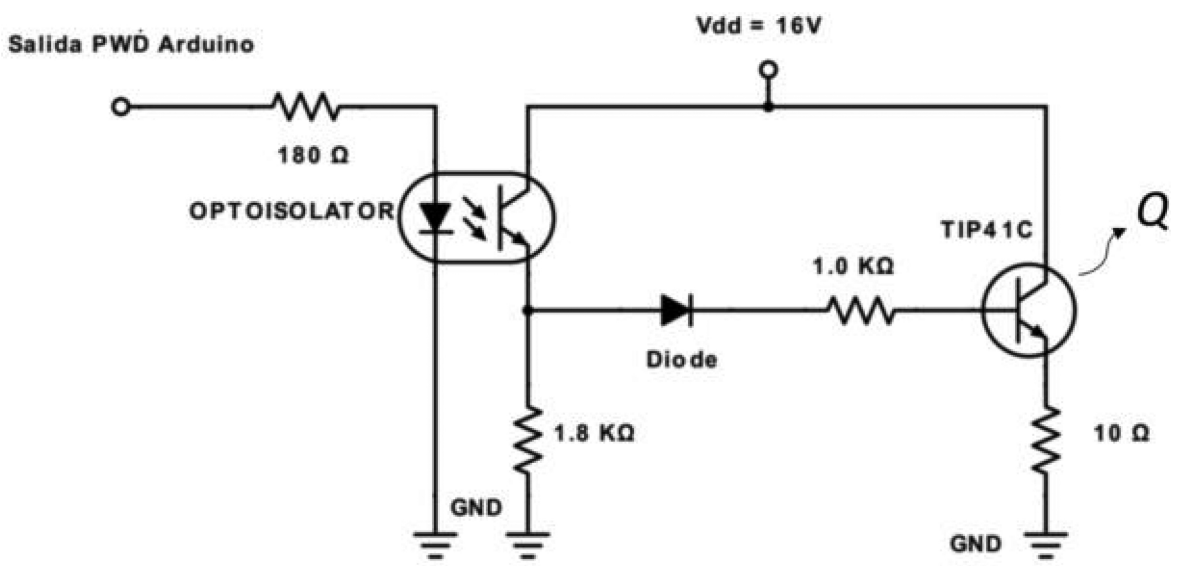

Figura 4: Circuito de calentamiento de la barra.

- Se inicia el programa Scilab. Desde el menú "File/ Execute"se ejecuta el archivo loader.sce existente en la carpeta de instalación del toolbox Arduino for Xcos. Este ejecutable carga los nuevos bloques de programación para el ambiente $X \cos$ de forma a poder realizar operaciones de lectura y escritura directamente desde, y hacia, los pines de la placa.

- La figura (5) muestra el programa de bloques de $\mathrm{X} \cos$ que controla el circuito de alimentación (salida PWM) y la adquisición de los voltajes de los sensores. De la figura se tiene:
- La placa está comunicada mediante el puerto COM 4.

- Se realiza una adquisición de datos durante 5400 segundos, realizando una muestra cada 0.1 segundo.

- La adquisición de los tres sensores se realiza mediante bloques de entrada analógica (pines 0,1 y 2). Los datos ingresan con una representación digital de 10 bits (desde 0 para un voltaje de 0V a 1023 para un voltaje de $5 \mathrm{~V})$, siendo que los sensores de temperatura

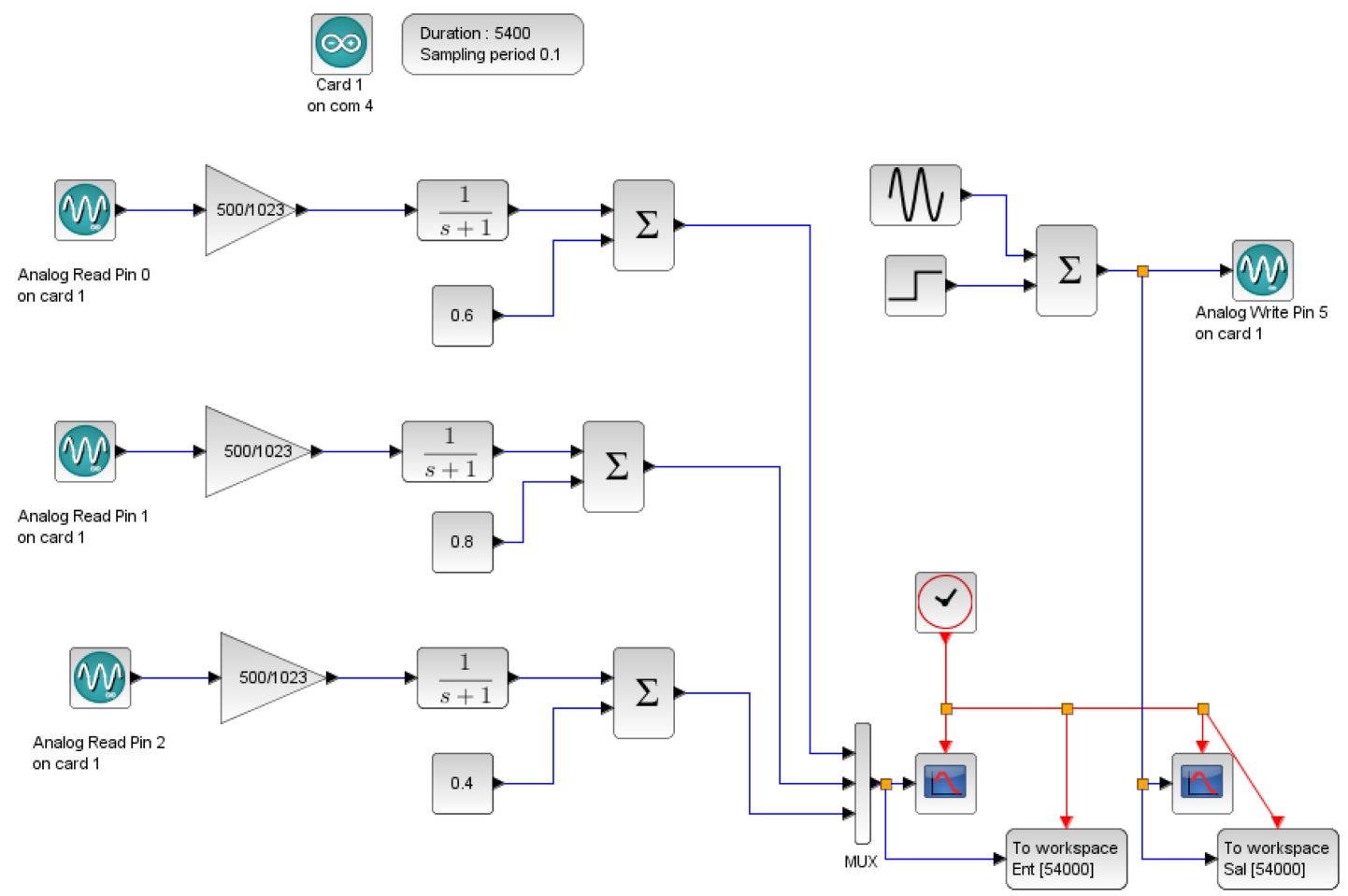

Figura 5: Programa de bloques de Xcos. Los bloques a la izquierda (las tres entradas analógicas del Arduino) leen los datos de los sensores. El bloque de ganancia, traduce voltaje a un valor de temperatura. Luego el filtro se usa para eliminar el ruido, y su salida va sumada con un offset, el cual se introduce para calibrar los sensores. Las salidas se grafican y se guardan en los bloques (llamados "To workspace"). En la salida PWM del Arduino, se suma un escalón de continua de altura dada y una sinusoidal de amplitud y frecuencia dadas. (Se puede ingresar solamente un escalón, dando cero al valor de amplitud de la sinusoide). 
producen voltajes desde $0 \mathrm{~V}$ para una temperatura de $2{ }^{\circ} \mathrm{C}$ a $1,5 \mathrm{~V}$ para una temperatura de $100^{\circ} \mathrm{C}$. Los bloques de ganancia 500/1023 realizan el ajuste necesario para la conversión de los datos a temperaturas en grados Celsius. Posteriormente la señal es filtrada mediante un filtro analógico de primer orden con constante de tiempo de 1 segundo. Previo al experimento se calibra los bloques de constantes anteriores al sumador para que las señales provenientes de todos los sensores reflejen la misma temperatura ambiente (constantes de calibración).

- El pin 5 de la placa está configurado como una salida analógica y se le entrega valores entre 0 y 255 (salida de 8 bits). De esta forma es posible tener una señal PWM en el pin tal que el valor medio varíe de $0 \mathrm{~V}$ a $5 \mathrm{~V}$.

\section{Análisis de la propagación de calor}

En esta sección se estudia de propagación de calor en una barra mediante soluciones analíticas en régimen permanente en dos casos específicos (entrada de temperatura en escalón y entrada sinusoidal), y transitorios utilizando un modelo que utiliza sistemas eléctricos discretos acoplados representado por $N$ elementos de circuitos $\mathrm{RC}$ (resistencia-capacitor).

\subsection{Ecuación de propagación de calor}

Si se considera una barra metálica homogénea con una longitud $L$ mucho mayor que su ancho $l$ y espesor $e$ $(L \gg l, e)$, la propagación será relevante principalmente en una única dimensión. En este caso, la ecuación para la variación de temperatura $T(x, t)$ en derivadas parciales que gobierna la propagación de calor es del tipo parabólico 15. Esta se basa en la ley de conducción de Fourier y la ley de convección entre la barra y el aire circundante, y viene dada por

$$
\frac{k}{\rho c} \frac{\partial^{2} T}{\partial x^{2}}-\frac{h P}{A \rho c}\left(T-T_{a m b}\right)=\frac{\partial T}{\partial t}
$$

siendo $T_{a m b}$ la temperatura ambiente del aire circundante.

Las constantes físicas que aparecen son:

- $k$ es la conductividad térmica,

- $c$ el calor especifico,

- $\rho$ la densidad y

- $h$ el coeficiente de convección entre la superficie del aluminio y el aire,

siendo las magnitudes geométricas consideradas

- el perímetro $P=2(l+e) \mathrm{y}$

- el área de sección $A=l e$.
Esta ecuación se puede expresar en la variable

$$
u(x, t)=T(x, t)-T_{a m b},
$$

quedando de la forma

$$
\frac{\partial^{2} u}{\partial x^{2}}-m^{2} u=\frac{1}{\alpha} \frac{\partial u}{\partial t}
$$

siendo

- $\alpha=\frac{k}{\rho c}$ el coeficiente de difusividad y

- $m=\sqrt{\frac{h P}{k A}}$ el coeficiente afín asociado a la convección.

\subsection{Solución en régimen estacionario para una entrada escalón}

En este primer caso se analiza el régimen permanente $(t \rightarrow \infty)$ considerando una entrada de temperatura del tipo escalón en el extremo de la barra. Siendo así, el término derecho de la ecuación (3) se anula dando

$$
\frac{\partial^{2} u}{\partial x^{2}}-m^{2} u=0
$$

Esta ecuación tiene una solución analítica dada por 15

$$
u(x)=A e^{-m x}+B e^{m x},
$$

donde $A, B$ son constantes que dependen de las condiciones de frontera. Para una entrada escalón de altura $u_{i n}$ las condiciones de frontera en el extremo conectado al transistor $(x=0)$ será

$$
u(0, t)=u_{i n}=A+B=T_{i n}-T_{a m b},
$$

siendo que para el otro extremo $(x=L)$,

$$
\frac{\partial u}{\partial x}+\frac{h}{k} u=0
$$

Considerando $\beta=\frac{h}{k}$, se tiene que

$$
\begin{aligned}
A & =\frac{u_{i n} e^{m L}\left(1+\frac{\beta}{m}\right)}{2\left(\frac{\beta}{m} \sinh (m L)+\cosh (m L)\right)}, \\
B & =u_{i n}-A .
\end{aligned}
$$

Como se puede observar, el estudio de la entrada de escalón en régimen estacionario nos permite calcular el coeficiente de convección $h$ en forma independiente del coeficiente de difusividad $\alpha$, como realizado en la experiencia de la subsección 4.1 .

\subsection{Solución en régimen estacionario para una entrada sinusoidal}

Por ser este un sistema lineal, para una entrada sinusoidal de frecuencia angular $\omega$,

$$
u(x=0, t)=u_{i n} \cos (\omega t),
$$


en el extremo de la barra, la solución en estado estacionario para cualquier punto debe ser una sinusoidal de la misma frecuencia $\omega[16$. Siendo así la solución se puede representar como

$$
u(x, t)=\Re\left\{e^{i \omega t}\left(\hat{A} e^{-(a+i b) x}+\hat{B} e^{(a+i b) x}\right)\right\} .
$$

Sustituyendo esta ecuación en (3) se obtiene

$$
\begin{aligned}
a & =\frac{1}{\sqrt{2 \alpha}}\left(\sqrt{\omega^{2}+\alpha^{2} m^{4}}+\alpha m^{2}\right)^{1 / 2}, \\
b & =\frac{\omega}{2 a \alpha} .
\end{aligned}
$$

Las funciones complejas $\hat{A}(\omega)$ y $\hat{B}(\omega)$ se encuentran usando condiciones de frontera similares a las utilizadas para la entrada escalón (pero en este caso con constantes complejas). Para $x=0$ se tiene que $\hat{A}+\hat{B}=u_{i n}$, y para $x=L$ la condición viene dada por la ecuación (7).

La ecuación (11) puede ser reescrita de la forma concisa

$$
u(x, t)=M(\omega, x) u_{i n} \cos (\omega t+\phi(\omega, x))
$$

siendo $M(\omega, x)$ la ganancia en amplitud y $\phi(\omega, x)$ el desfasaje con respecto a la entrada 10, , como se ilustra en la Figura (6).

Nótese que en el límite de $\omega \rightarrow 0$, se tiene que $a \rightarrow m$ y $b \rightarrow 0$, de donde la solución en régimen del escalón ecuación (5) es una caso particular de la ecuación (11).

\subsection{Velocidad de propagación de información: velocidad de grupo.}

La ecuación (3) que gobierna la propagación de calor es del tipo parabólico [15], lo que hace que la rapidez de propagación del calor sea, en principio, infinita. Este problema es conocido desde el tiempo de Maxwell [17. e incompatible desde el punto de vista de la teoría de la relatividad, siendo que experimentalmente la velocidad de propagación es siempre finita 18.

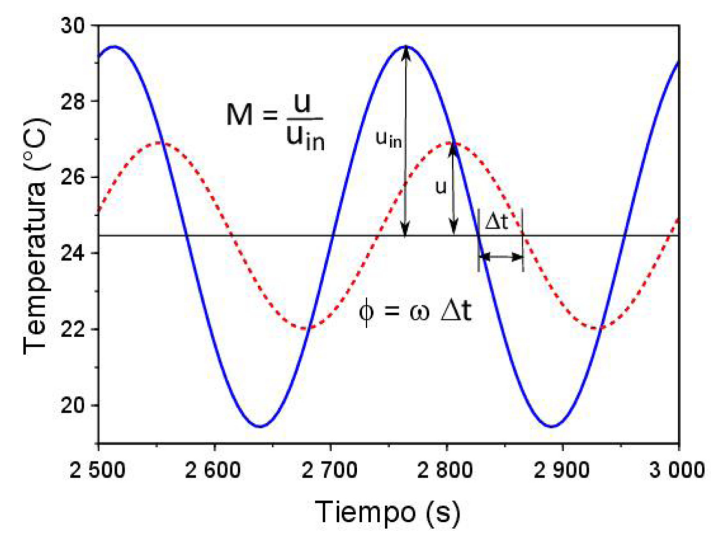

Figura 6: Salida sinusoidal en régimen en un punto $x$ de la barra (linea punteada, amplitud $u$ ) para una entrada sinusoidal (línea sólida, amplitud $u_{i n}$ ) y una cierta frecuencia $\omega$. Se ilustra la ganancia en amplitud $M$ y el defasaje $\phi$ con respecto a la entrada.
Esto puede ser explicado tomando en cuenta que para que exista transporte de información, o sea, que se pueda percibir un cambio de temperatura en un punto cualquiera de la barra dado un cambio en un extremo, debe superarse cierto umbral de temperatura en ese punto. El tiempo de demora en llegar a este umbral debido al transitorio, hace que la velocidad de la información sea finalmente finita [19] [20]. Para remediar este problema varias alternativas a las ecuaciones anteriores fueron propuestas [18] 21] 22].

Por otro lado, para bajas frecuencias se puede estimar la velocidad de propagación usando las ecuaciones anteriores, ya que son una buena aproximación de la realidad [8]. Similar a una onda de luz en medio dispersivo, la velocidad de grupo que se define como $v_{g}=\frac{d \omega}{d b}, \mathrm{y}$ es una buena estimación de la velocidad de propagación. Esta velocidad se deduce de la ecuación (13), dando

$$
v_{g}=\sqrt{8 \alpha} \frac{\delta\left(\delta^{2}+\alpha^{2} m^{4}\right)+2 \alpha m^{2} \delta^{2}}{\left(\delta+\alpha m^{2}\right)^{1 / 2}\left(2 \alpha m^{2} \delta+\delta^{2}+\alpha^{2} m^{4}\right)}
$$

siendo $\delta(\omega)=\sqrt{\omega^{2}+\alpha^{2} m^{4}}$.

Notese que para $\omega \rightarrow 0$, o sea, para el caso de una entrada escalón (temperatura constante en régimen estacionario), $v_{g}=2 \alpha m$. Por otro lado si no se considera nula la convección $h=0$, entonces la velocidad de grupo es $v_{g}=2 \sqrt{2} \alpha \omega$ como visto en 23 .

\subsection{Solución del régimen transitorio: modelo discreto de resistencias y capacitores acoplados.}

La solución de la ecuación (3) es en general compleja. Si bien existen soluciones analíticas de esta ecuación basadas en separación de variables [6] [24], se obtiene finalmente una serie de Fourier con infinitos coeficientes, que tienen que cumplir con condiciones iniciales y de frontera. En este trabajo se ha optado por resolver este problema utilizando un modelo equivalente eléctrico a la trasmisión de calor, por ser esta solución sencilla y fácil de comprender para estudiantes de grado de ingeniería o ciencias básicas.

Es posible realizar un símil eléctrico para el flujo de calor utilizando $N$ celdas, donde cada celda tiene una longitud $\Delta x=\frac{L}{N}$ (discretización espacial de la barra en $N$ elementos). En este caso, se puede considerar una analogía física entre el flujo de calor $q=\frac{\delta Q}{d t}$ (donde $q$ es el calor transferido por unidad de tiempo) y el flujo de carga eléctrica o corriente $I$. A su vez, la temperatura $T_{i}(t)$ en cada elemento de la barra $(i=1 \ldots N)$ es análoga al potencial eléctrico $V_{i}(t)$. Así, como se muestra en la figura 7, los componentes eléctricos del circuito serían análogos a

- $R_{k}=\Delta x /(k A)$, resistividad térmica de conducción,

- $R_{h}=(h P \Delta x)^{-1}$, resistividad térmica de convección,

- $C=c A \rho \Delta x$ la capacidad calorífica. 


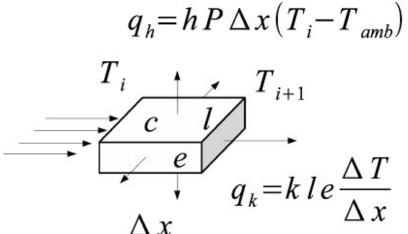

(a)

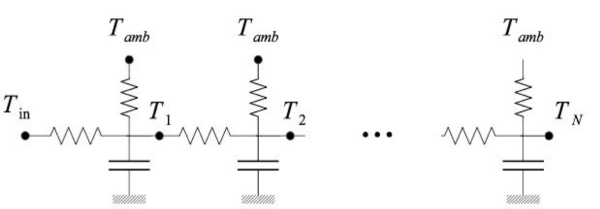

(c)

Figura 7: Analogía de sistema de trasmisión de calor (a) mediante un símil eléctrico (b). Sistema completo con $N$ elementos (c).

Si se considera las variaciones de pequeña señal respecto a una temperatura ambiente fija $T_{a m b}^{*}$, es posible considerar el sistema con una única señal de entrada $u_{i n}=T_{i n}-T_{a m b}^{*}$ (análogo a la subsección 3.1, resultando en un sistema de ecuaciones diferenciales de primer orden

$$
\dot{\mathbf{X}}=A \mathbf{X}+B \mathbf{U}
$$

siendo

$A=\left[\begin{array}{cccccc}\gamma & \frac{1}{R_{k} C} & 0 & \cdots & 0 & 0 \\ \frac{1}{R_{k} C} & \gamma & \frac{1}{R_{k} C} & \cdots & 0 & 0 \\ 0 & \frac{1}{R_{k} C} & \gamma & \cdots & 0 & 0 \\ \vdots & \vdots & \vdots & \vdots & \vdots & \vdots \\ 0 & 0 & \cdots & 0 & \frac{1}{R_{k} C} & \hat{\gamma}\end{array}\right], B=\left[\begin{array}{c}\frac{1}{R_{k} C} \\ 0 \\ 0 \\ \vdots \\ 0\end{array}\right]$,

(17)

$\gamma=\frac{-2 R_{h}+R_{k}}{R_{k} R_{h} C}$ y $\hat{\gamma}=\frac{-R_{h}+R_{k}}{R_{k} R_{h} C}$, donde

$$
\mathbf{X}=\left[\begin{array}{llll}
u_{1} & u_{2} & \ldots & u_{N}
\end{array}\right]^{T} \in \mathbb{R}^{N}
$$

con $u_{i}$ la temperatura del punto $i$-ésimo de la barra respecto a la temperatura $T_{a m b}^{*}$ y $\mathbf{U}=u_{i n}$ la temperatura en el primer sensor.

\section{Experiencias}

\subsection{Determinación del coeficiente $h$ de convección}

Para determinar el coeficiente de convección, con una entrada constante en el comando del transistor se caliente el extremo de la barra hasta que las temperaturas en todos los sensores lleguen a régimen.

En la figura (8) se muestra el resultado de cuatro ensayos con temperaturas de régimen diferentes en el transistor, ilustrando las medidas tomadas en los tres sensores y las respectivas curvas analíticas resultantes dadas por la ecuación (5). La figura (9) muestra las curvas normalizadas, en la cual se observa la dispersión en cada uno de los tres sensores.

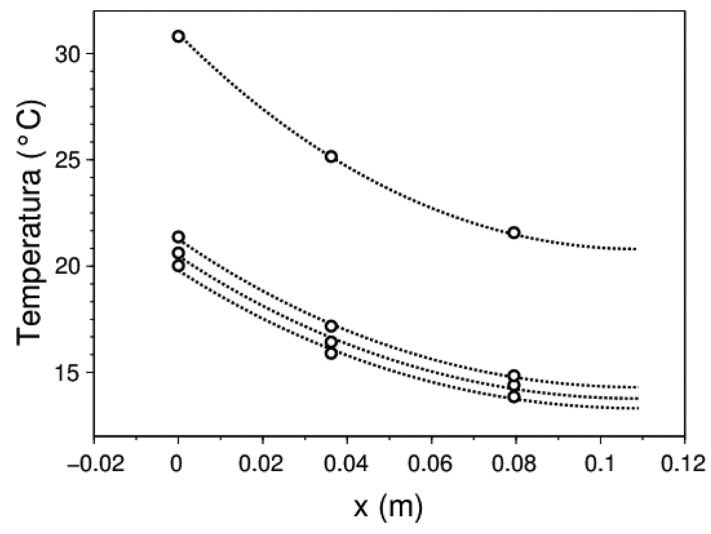

Figura 8: Soluciones analíticas para las temperaturas en toda la barra (líneas punteadas) correspondientes a la solución de la ecuación de calor para cuatro ensayos y las respectivas medidas de los tres sensores en cada caso (círculos).

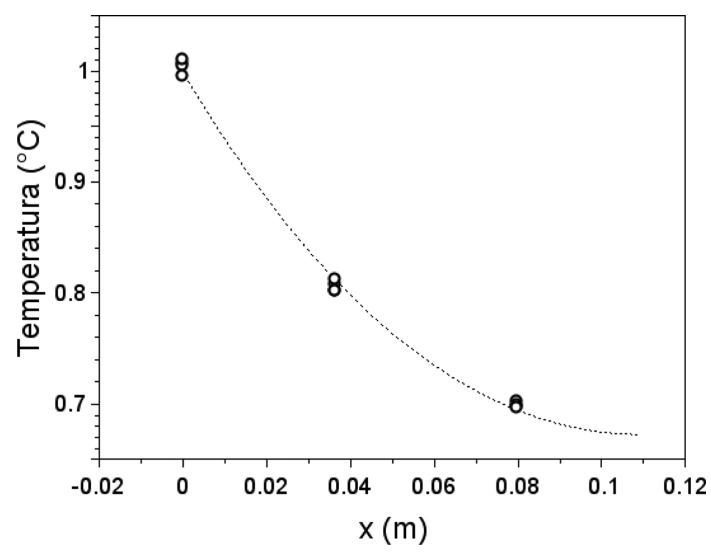

Figura 9: Solución analítica normalizada

Para el ajuste de las curvas se ha realizado una optimización (mínimos cuadrados) para la obtención experimental del coeficiente de convección, resultado en $h=14,4 \pm 1,3\left(\mathrm{~W} / \mathrm{m}^{2} \mathrm{~K}\right)$.

\subsection{Coeficiente de difusividad $\alpha$ y corrección debido a los sensores.}

Con la finalidad de modelar los transitorios de calor en la barra se utiliza un sistema de ecuaciones diferenciales de orden $N=74$ (cantidad de particiones de la barra) del RC tipo análogo como el indicado en la subsección 3.5

Como la primera señal de temperatura conocida es la medida del primer sensor, se realiza el modelo utilizando esta señal como entrada, o sea, $u_{i n}$, determinando así la temperatura las otras posiciones de la barra, y en particular en la posición de los dos sensores restantes.

En la realización de las experiencias se utiliza un valor constante en la alimentación del transistor, resultando en las señales ilustradas con color gris en la figura 10

Realizando la solución numérica con el valor estándar de $\alpha$ encontrado en la literatura, cuadro 1. se tiene como resultado que el transitorio de las temperaturas en los últimos sensores no coinciden con los resultados experimentales.

Observando los resultados anteriores se ha analizado que la diferencia podría deberse a efectos locales, ya que los sensores 


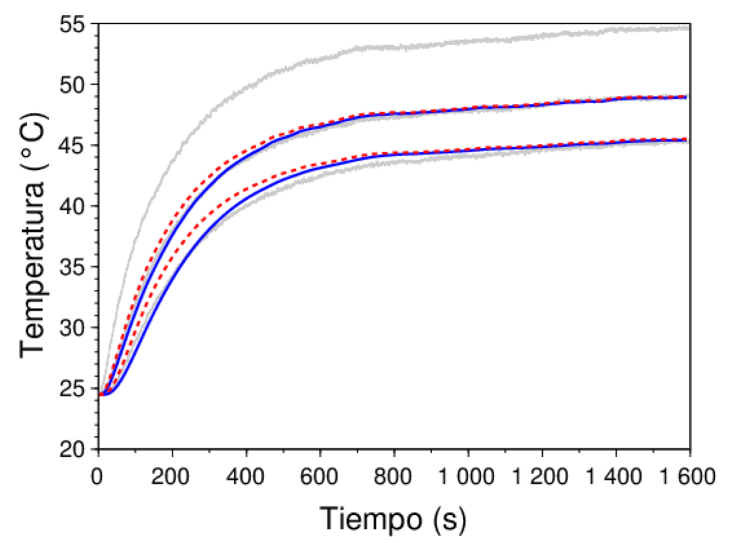

Figura 10: Variación de las temperaturas de los tres sensores (puntos grises) debido a aplicación de un valor medio constante en el transistor. Las curvas a trazos, y sólida, corresponden a las soluciones numéricas utilizando usando constantes estándar, y con correcciones locales en los sensores, respectivamente.

Cuadro 1: Constantes físicas del aluminio, 25]

\begin{tabular}{cccc}
\hline$k(W /(m K))$ & $\rho\left(K g / m^{3}\right)$ & $c(J /(K g K))$ & $\alpha\left(m^{2} / s\right)$ \\
\hline 209,3 & 2686 & 897 & $8,69 \times 10^{-5}$ \\
\hline
\end{tabular}

utilizados afectan la capacidad calorífica y la convección. Modificando localmente el modelo del sistema en la posición de los sensores, es posible realizar un ajuste de las constantes tal que se obtiene los resultados indicados los trazos sólidos de la figura 10 Los datos obtenidos en este caso implican un ajuste de $h_{S}=0,91 h$ (coeficiente de convección local) y $C_{S}=8 C$ (capacidad calorífica local).

\subsection{Solución de la entrada sinusoidal}

A modo de verificar el buen desempeño del modelo anterior, se excita el equipo con una entrada sinusoidal y se muestrea las temperaturas en los sensores. Los datos del primer sensor son utilizados como entrada al modelo RC y se obtiene las señales de salida en las posiciones de los posteriores sensores. La figura 11 ilustra el resultado de la comparación entre los datos reales y el calculado numéricamente (considerando la temperatura ambiente).

Utilizando este modelo es posible, para el sistema en régimen sinusoidal, calcular la ganancia en amplitud $M(\omega, x)$ y el defasaje $\phi(\omega, x)$ (subsección 3.3 para cada punto de la barra y frecuencia de trabajo. Las figuras 12 y 13 ilustran este resultado. De las figuras se observa que para frecuencias $\omega \rightarrow 0$ la curva de ganancia es la ilustrada en la figura 9 .

\section{Conclusiones}

En este artículo se presenta la construcción de un equipo de bajo costo para el estudio de la propagación de calor en una barra metálica, utilizando la tecnología Arduino y el ambiente $X c o s$ del Scilab. Uno las principales ventajas de la propuesta es que puede ser fácilmente adaptada para otros dispositivos destinados a laboratorios de enseñanza de la física.

Como ejemplo de uso se ha analizado la propagación de calor en una barra de aluminio, incluyendo el efecto de la

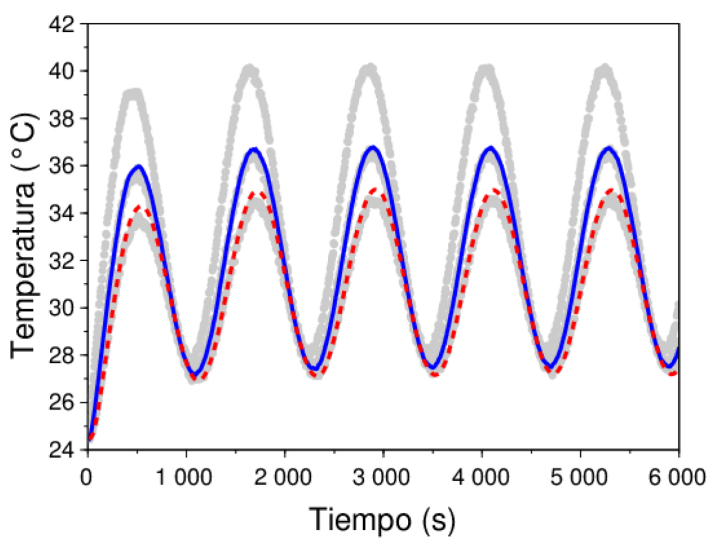

Figura 11: Temperatura experimental de los tres sensores para una entrada sinusoidal (puntos grises), junto con las curvas de la solución numérica, segundo sensor (linea entera azul), tercer sensor(linea a trazos roja).

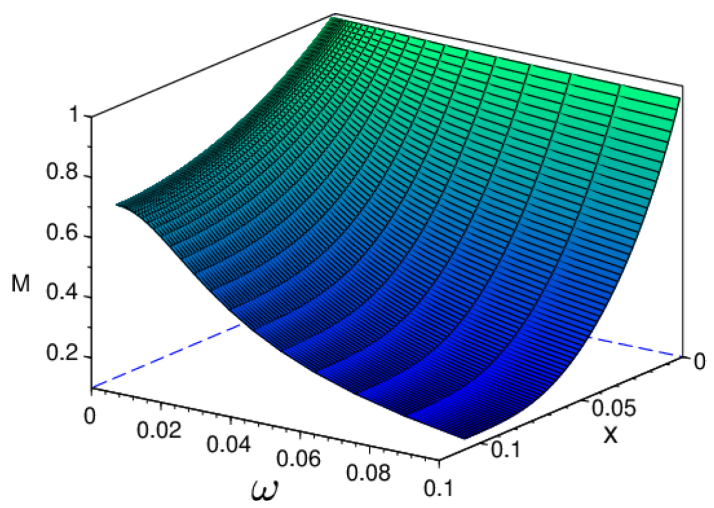

Figura 12: Ganancia de temperatura en régimen sinusoidal $M(\omega, x)$, en función de la frecuencia $\omega$ y la posición en la barra $x$.

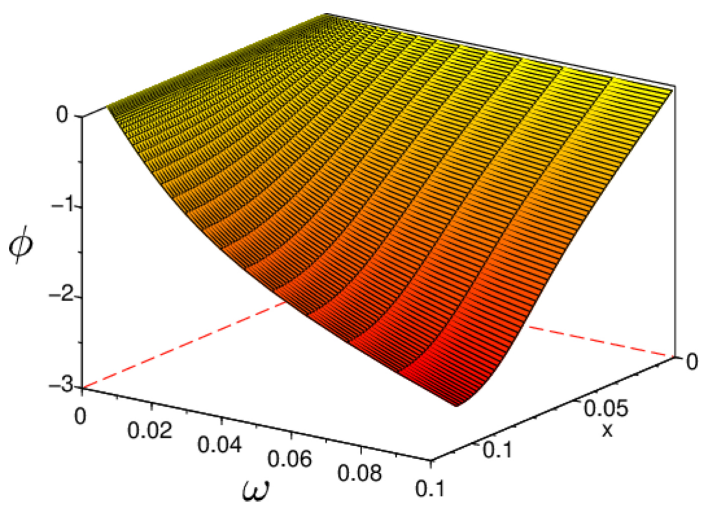

Figura 13: Defasaje de la temperatura en régimen sinusoidal $\phi(\omega, x)$, en función de la frecuencia $\omega$ y la posición en la barra $x$.

convección. Mediante experimentos se ha determinado la constante de convección de calor entre el aluminio y el aire para el caso particular de la geometría de la barra utilizada.

Con la utilización de un modelo discreto de la barra basado en un sistema de ecuaciones diferenciales ordinarias de primer orden se ha estudiado el comportamiento transitorio de la 
propagación de calor. Se ha verificado que, en la posición de los sensores, el sistema debería ser localmente corregido para ajustar con los datos experimentales.

\section{Agradecimientos}

Agradecemos al Prof. Martín Monteiro de la Universidad ORT Uruguay por las sugerencias realizadas en la redacción de este artículo.

\section{Referencias}

[1] https://www.arduino.cc/.

[2] C. Llamas Bello, J. Vegas, M. González Rebollo y M. González Delgado, Papers in Physics 10, 100004 (2018).

[3] G. Calderón, J.H. Muñoz y J.Y. Rivera, Revista Brasileira de Ensino de Física 40, e2402 (2018).

[4] F. Bouquet, J. Bobroff, M. Fuchs-Gallezot y L. Maurines, American Journal of Physics 85, 216 (2017).

[5] A. Marzo, A. Barnes y B.W. Drinkwater, Review of Scientific Instruments 88, 085105 (2017).

[6] J. Brody y M. Brown, American Journal of Physics 85, $582(2017)$

[7] R. Herman, A. Ballowe y J. Ashley, The Physics Teacher 55, 488 (2017).

[8] A. Salazar, European Journal of Physics 27, 134 (2006).

[9] http://s4a.cat/.

[10] http://blog.ardublock.com/.

[11] R. Christiansen, F. Hanna, E. Aguero y N. Pereyra, Revista de enseñanza de la fisica 28, 23 (2016).

[12] J. Lesteiro-Tejeda, D. Hernández-Delfín y A. BatistaLeyva, Revista Cubana de Física 34, 120 (2017).

[13] www.scilab.org.

[14] https://atoms.scilab.org/toolboxes/arduino.

[15] T.L. Bergman, F.P. Incropera, D.P. DeWitt y A. Lavine, Fundamentals of Heat and Mass Transfer (John Wiley \& Sons, Hoboke, 2011), $7^{\mathrm{a}} \mathrm{ed}$.

[16] K. Ogata, Modern Control Engineering (Prentice Hall PTR, Upper Saddle River, 2001), $4^{\mathrm{a}}$ ed.

[17] D.D. Joseph y L. Preziosi, Rev. Mod. Phys. 61, 41 (1989).

[18] P. Ván, Communications in Applied and Industrial Mathematics 7, 150 (2016).

[19] V. Bertola y E. Cafaro, Physics Letters A 372, 1 (2007).

[20] I. Shnaid, International Journal of Heat and Mass Transfer 46, 3853 (2003).

[21] B. Soma, C. Baláz, F. Tamás, G. Gyula, G. Ákos, K. Róbert, P. Ván y V. József, Journal of Non-Equilibrium Thermodynamics 41, 41 (2015).

[22] M. Makai, Europhysics Letters 96, 40010 (2011).

[23] E. Marín, L.S. Vaca-Oyola y O. Delgado-Vasallo, Revista mexicana de física E 62, 1 (2016).

[24] Y. Yener y S. Kakac, Heat Conduction (CRC Press, Boca Raton, 2008).

[25] E. MacCormack, A. Mandelis, M. Munidasa, B. Farahbakhsh y H. Sang, Int J Thermophys 18, 221 (1997). 